\title{
Further identification of a 140bp sequence from amid intron 9 of human FMR1 gene as a new exon
}

\author{
Wen-jing Yang ${ }^{1,2+}$, Ai-zhen Yan ${ }^{1 \dagger}$, Yong-jun Xu ${ }^{1 \dagger}$, Xiao-yan Guo ${ }^{1,3}$, Xian-guo Fu ${ }^{1,4}$, Dan Li ${ }^{1}$, Juan Liao ${ }^{1,5}$,
} Duo Zhang ${ }^{1}$ and Feng-hua Lan ${ }^{1 *}$ (i)

\begin{abstract}
Background: The disease gene of fragile $X$ syndrome, FMR1 gene, encodes fragile $X$ mental retardation protein (FMRP). The alternative splicing (AS) of FMR1 can affect the structure and function of FMRP. However, the biological functions of alternatively spliced isoforms remain elusive. In a previous study, we identified a new 140bp exon from the intron 9 of human FMR1 gene. In this study, we further examined the biological functions of this new exon and its underlying signaling pathways.

Results: qRT-PCR results showed that this novel exon is commonly expressed in the peripheral blood of normal individuals. Comparative genomics showed that sequences paralogous to the 140 bp sequence only exist in the genomes of primates. To explore the biological functions of the new transcript, we constructed recombinant eukaryotic expression vectors and lentiviral overexpression vectors. Results showed that the spliced transcript encoded a truncated protein which was expressed mainly in the cell nucleus. Additionally, several genes, including the BEXI gene involved in mGluR-LTP or mGluR-LTD signaling pathways were significantly influenced when the truncated FMRP was overexpressed.

Conclusions: our work identified a new exon from amid intron 9 of human FMR1 gene with wide expression in normal healthy individuals, which emphasizes the notion that the AS of FMR1 gene is complex and may in a large part account for the multiple functions of FMRP.
\end{abstract}

Keywords: FMR1, FMRP, FXS, Alternative splicing, Alternative exon

\section{Background}

Fragile X syndrome (FXS) is a common form of inherited intellectual disability, affecting 1/7000 females and 1/4000 males. Apart from mild to severe intellectual disability, individuals with FXS often exhibit autism-like behavior, including social anxiety, attention deficit, mood disturbance, and

\footnotetext{
* Correspondence: fhlan@xmu.edu.cn

${ }^{+}$Wen-jing Yang, Ai-zhen Yan and Yong-jun Xu contributed equally to this work.

'Department of Clinical Genetics and Experimental Medicine, 900th Hospital of the Joint Logistics Force, Xiamen University School of Medicine, 156

Xi'erhuanbei Road, Fuzhou City, Fujian Province 350025, People's Republic of China

Full list of author information is available at the end of the article
}

sleep disorders. FXS patients also present other prominent physical symptoms, encompassing a long, narrow face with large protruding ears, flat feet, eye-gaze avoidance, and macroorchism. Pathogenically, when the trinucleotide CGG repeat in the $5^{\prime}$ untranslated region of the fragile $\mathrm{X}$ mental retardation 1 (FMR1) gene expands to more than 200, DNA methylation and transcriptional silencing of $F M R 1$ will be occur, leading to the loss of FMR1 gene product, that is, fragile X mental retardation protein (FMRP) [1]

In mammals, full-length FMR1 gene sequence encodes a $71 \mathrm{kDa}$ FMRP. FMRP has three motifs, including two $\mathrm{K}$ homology domains (KH1 and $\mathrm{KH} 2$ ) encoded by exons 7-9 and exons 9-13, respectively, and the arginine-

(c) The Author(s). 2020 Open Access This article is licensed under a Creative Commons Attribution 4.0 International License, which permits use, sharing, adaptation, distribution and reproduction in any medium or format, as long as you give appropriate credit to the original author(s) and the source, provide a link to the Creative Commons licence, and indicate if changes were made. The images or other third party material in this article are included in the article's Creative Commons licence, unless indicated otherwise in a credit line to the material. If material is not included in the article's Creative Commons licence and your intended use is not permitted by statutory regulation or exceeds the permitted use, you will need to obtain permission directly from the copyright holder. To view a copy of this licence, visit http://creativecommons.org/licenses/by/4.0/. The Creative Commons Public Domain Dedication waiver (http://creativecommons.org/publicdomain/zero/1.0/) applies to the data made available in this article, unless otherwise stated in a credit line to the data. 
glycine-glycine (RGG) box encoded by exons $15-16$, that mediates RNA or protein interaction. FMRP also contains a nuclear localization signal (NLS) and a nuclear export signal (NES). Additionally, numerous studies showed that FMRP has agenet domains at $\mathrm{N}$ terminus to combine with methylated H3K9 chromatin [2] FMRP is also a selective mRNA-binding protein that regulates RNA transcription, splicing, and cell apoptosis [3] It is estimated that FMRP can bind more than 5\% mRNA in cells. Yeast three-hybrid assay and microarray have detected as many as 400 potential mRNAs related to FMRP [4] FMRP is widely expressed and especially abundant in brain, playing a critical role in synaptic plasticity and neurological signaling pathways as a translational repressor. Among them, metabotropic glutamate receptor-long-term depression (mGluR-LTD) is one of the most common pathways that can regulate the $\alpha$ amino-3-hydroxy-5-methyl-4-isoxazolepropionic acid receptor (AMPAR) internalization and local protein synthesis to prevent deficits in synaptic plasticity [5] In addition, mGluR-long-term potentiation (mGluR-LTP), phosphorylation of FMRP, gamma-aminobutyric acid (GABA), and dopamine receptors (DA) signaling pathways are all required for the formation of normal neurological function [6]

Mature transcripts from FMR1 gene have multiple alternatively spliced isoforms in different organs. The most common ways of alternative splicing (AS) are the inclusion or exclusion of exons 12 and 14, and the selection of splice acceptor sites on exons 15 and 17 [7] FMR1 has been reported to produce more than 20 FMRP isoforms with various structures and functions. Different FMRP isoforms may be involved in various signal transduction pathways, implying their significant biological functions. With the incidence of 50 to $75 \%$ of in central nervous system, alternatively spliced genes have important influence on synaptic plasticity [8] ion channel activity [9] the genesis of dendritic spines [10] and the release of neurotransmitters [11] FMR1 is an example of key gene that produces various spliced transcripts in human brain. However, the exact number of alternatively spliced isoforms in various tissues and cells and their specific biological functions are still poorly understood.

Previously, we and others reported a novel $140 \mathrm{bp}$ exon when detecting alternatively spliced transcripts from FMR1 gene $[12,13]$ This $140 \mathrm{bp}$ sequence comes from intron 9 of human FMR1 gene and introduces a premature stop codon in the resulting mRNAs. Bioinformatics analysis shows that the novel sequence has canonical splicing signals, implying that its potential as an alternative exon. Furthermore, qRT-PCR showed the exon can be detected in mature mRNA molecules of the peripheral blood from non-FXS individuals. Using eukaryotic expression vector and lentiviral vector for truncated FMRP analysis, we determined that the inclusion of the novel $140 \mathrm{bp}$ sequence leads to a truncated protein with altered subcellular distribution. RNA microarray analysis on cells overexpressing the truncated FMRP revealed a group of differentially expressed genes that might contribute to the FMRP signaling pathways. Our work emphasizes the notion that the AS of FMR1 gene is considerably more complex than what we have realized, and such complexity may in a large part account for the multiple functions of FMRP.

\section{Results}

Comparative genomics shows that sequences paralogous to the $140 \mathrm{bp}$ sequence only exist in the primate genomes To determine the relative abundance of FMR1 gene expression in the peripheral blood of normal non-FXS individuals, real-time reverse transcriptase PCR (qRT-PCR) was carried out using specific primers (Supplementary Tab. S1) aiming at the $140 \mathrm{bp}$ sequence. Box plot revealed that the first quartile ( $25 \%$ percentile) of $140 \mathrm{bp}$ mRNA expression was 0.026 , the second quartile (median) was 0.042 , and the third quartile (75\% percentile) was 0.069 . Among them, the minimum value of $140 \mathrm{bp}$ mRNA expression was 0.0187 , and the maximum value was 45.960 (Fig. 1). These results indicated that FMR1 mRNA containing the $140 \mathrm{bp}$ sequence exists ubiquitously in the peripheral blood of normal healthy individuals.

We further searched the genomes of other species for sequences similar to this new spliced variant. Genomes of FMR1 gene from diverse species were obtained and submitted to DANMAN software. The results showed that primates, such as Homo sapiens (GenBank: NC_ 000023), Macaca mulatta (GenBank: NC_012614), Pan troglodytes (GenBank: NC_006491), and Pongo abelii (GenBank: NC_007878), all have paralogues of the 140 bp sequence, although they are distributed in different introns of FMR1 gene, for example, in intron 9 of Homo

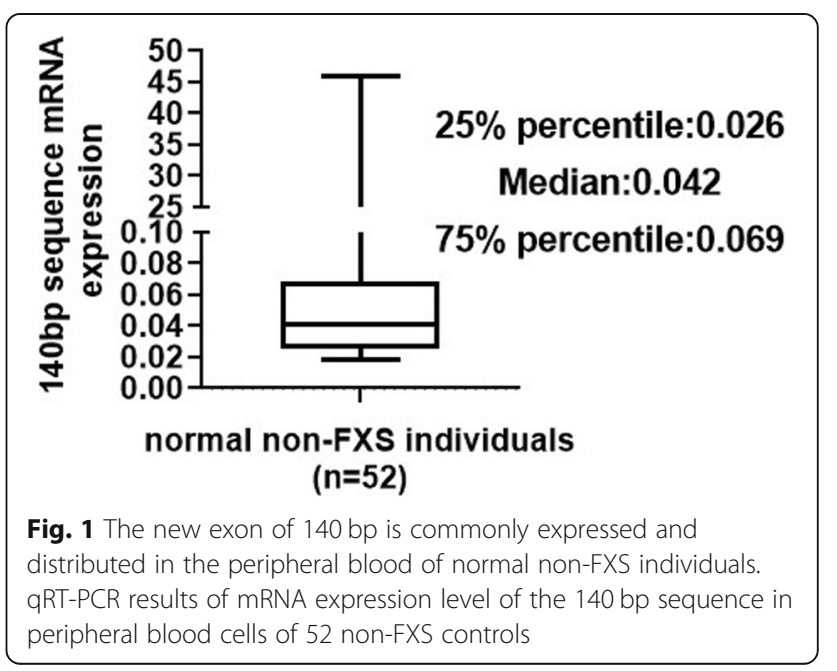


sapiens and Macaca mulatta and in intron 10 of Pan troglodytes and Pongo abelii (Supplementary Fig. S1). However, the $140 \mathrm{bp}$ sequence is absent in non-primates, implying that the $140 \mathrm{bp}$ sequence might be associated with the development and evolution of intelligence.

\section{FMR1 mRNA containing the 140 bp sequence can be translated into a truncated FMRP with altered cellular localization}

The splicing of the novel exon with exon 9 at the $5^{\prime}$ end and exon 10 at the $3^{\prime}$ end results in FMR1 mRNAs that can be translated into a $34 \mathrm{kDa}$ truncated FMRP containing 297 amino acid residuals, while lacking a large part of the carboxy-terminal domains, such as NES, the second KH domain, and RGG box (Fig. 2a). To identify the potential truncated protein and detect the expression of this newly alternative FMR1 transcript, we extracted the total proteins from peripheral blood cells of six normal non-FXS individuals and performed Western blot using a monoclonal antibody against the N-terminus of FMRP. The results showed that a protein band with a molecular weight of approximate $35 \mathrm{kDa}$, which is almost half the length of full-length FMRP could be seen in all six normal individuals (Fig. 2b).

To explore the subcellular localization of the end product from the new alternatively spliced FMR1 transcript, we constructed recombinant eukaryotic expression vectors with full-length coding sequence (pEGFPN2-fFMR1, full-length isoform) or coding fragment containing exons 1-9 together with the $140 \mathrm{bp}$ sequence (pEGFP-N2-tFMR1, truncated isoform), respectively. Western blot showed that HEK293T cells transfected by void plasmid, pEGFP-N2-fFMR1, and pEGFP-N2-tFMR1 all expressed a $71 \mathrm{kDa}$ endogenous FMRP. HEK293T cells transfected by pEGFP-N2-fFMR1 and pEGFP-N2tFMR1 expressed FMRP or truncated FMRP fused with EGFP, with molecular weights of 90 and $55 \mathrm{kDa}$, respectively (Fig. 3a). Immunofluorescence and quantitative representation of EGFP and FMRP positive cells revealed that the full-length FMRP was expressed mainly in the cytoplasm, whereas the truncated protein mainly existed in the cell nucleus, demonstrating an altered subcellular localization (Fig. 3b-d).

\section{FXS-related signal pathways are significantly influenced} when the truncated FMRP is overexpressed

RNA microarray analysis revealed 545 genes with altered expression (the top 20 up-regulated and down-regulated differentially expression genes are shown in Supplementary Tab. S2 and Tab. S3, respectively), among which $B E X 1$ (brain expressed X-linked 1) is the most significantly up-regulated gene, and GABRB3 (gamma-aminobutyric acid type A receptor beta3 subunit) is the most significantly down-regulated gene (Supplementary Tab. S2 and Tab. S3, respectively). To verify the microarray results, we selected 11 genes for qPCR, which demonstrated that 9 genes (BEX1, MAGE, MAGEB2, PNPLA4, PPP1R1A, GABRB3, NAP1L3, NAP1L2, and RGS-7) whose expression levels were altered to an extent similar to that shown in mRNA microarray analysis (Fig. 4a). Western blot showed that BEX1 gene was overexpressed in HEK293T cells stably transfected by pLEX-MCStFMR1 (Fig. 4b). Immunofluorescence and quantitative representation of FMRP and BEX1 positive cells revealed

A
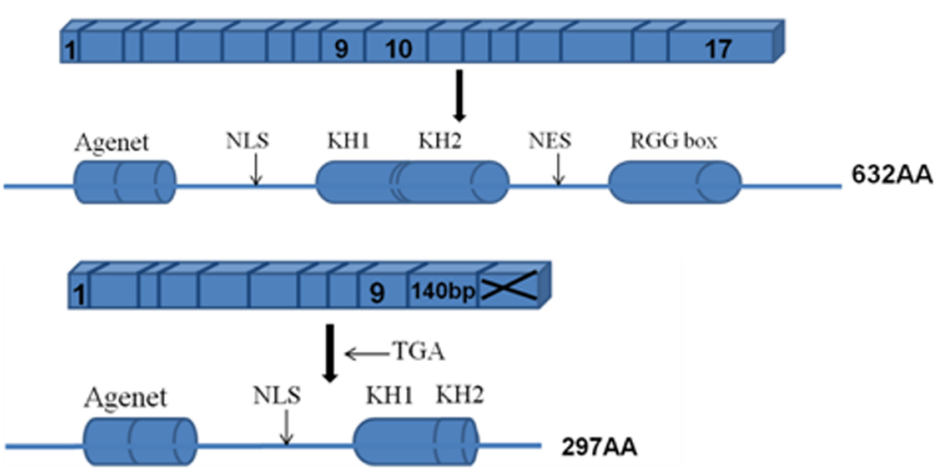

B

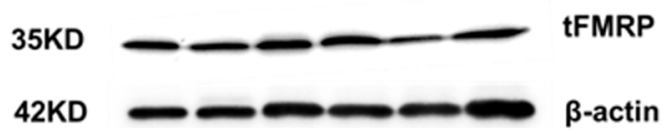

Fig. 2 The structure and expression of truncated FMRP protein in healthy individuals. a FMRP structure with coding exons (squares) and functional domains (columns). a: FMR1 gene containing full-length sequence encodes 632 amino acids; b: coding fragment containing exons 1-9 and the 140 bp sequence. $\mathbf{b}$ Western blots showing the expression of the new alternatively spliced FMR 1 transcript in peripheral blood cells of 6 healthy individuals. tFMRP: truncated FMRP 
A

\begin{tabular}{l|l|l|l} 
& 1 & 2 & 3 \\
$90 \mathrm{KD}$ & & & \\
$70 \mathrm{KD}$ & & & \\
$55 \mathrm{KD}$ & & & \\
\hline
\end{tabular}

pEGFP-N2-wFMRP

WFMRP

pEGFP-N2-tFMRP

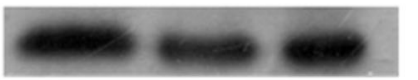

$\beta$ - actin

B

DAPI

EGFP

FMRP

Merge
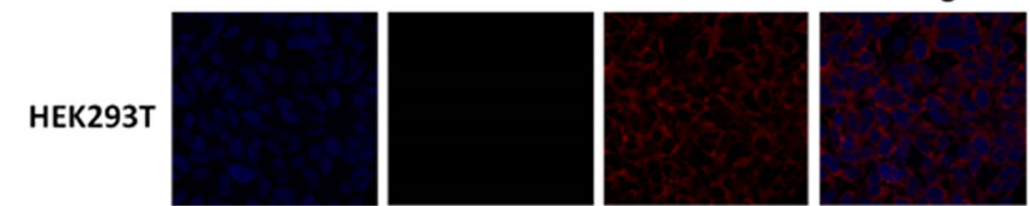

pEGFP-N2
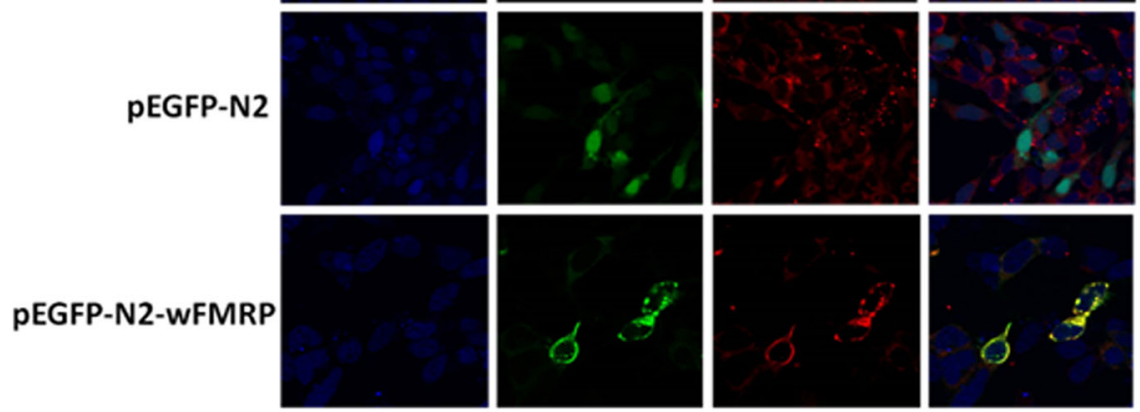

pEGFP-N2-tFMRP
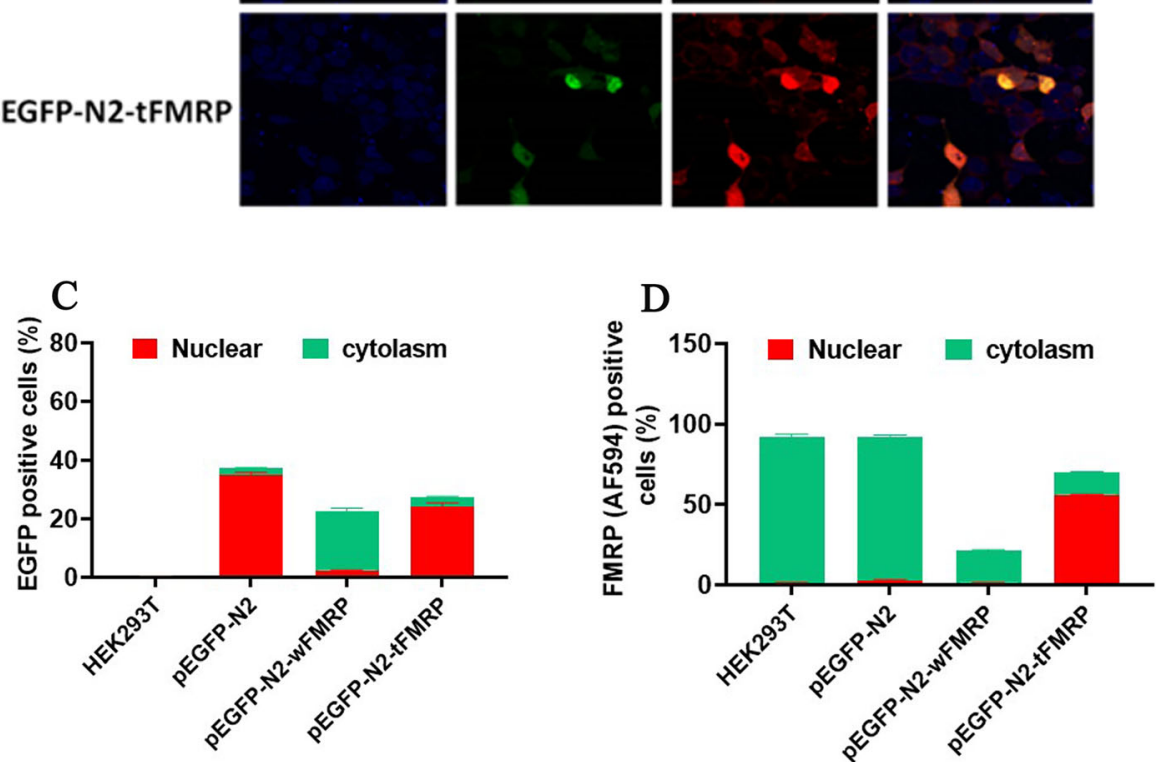

Fig. 3 The new spliced transcript containing the $140 \mathrm{bp}$ sequence is translated into a truncated FMRP protein with altered subcellular localization. a Western blots showing the expression of the wild type FMRP or the truncated FMRP in HEK293T cells. 1: untransfected HEK293T cells; 2:

HEK293T cells transfected by pEGFP-N2-tFMR1; 3: HEK293T cells transfected by pEGFP-N2-fFMR1. b Immunofluorescence showing the localization of the wild type FMRP or the truncated FMRP in HEK293T cells. HEK293T: untransfected HEK293T cells; pEGFP-N2: HEK293T cells transfected by the empty vector; pEGFP-N2-tFMR1: HEK293T cells transfected by pEGFP-N2-tFMR1; pEGFP-N2-fFMR1: HEK293T cells transfected by pEGFP-N2-fFMR1; DAPI: the solution suitable for nuclear staining; EGFP: vectors encoding the GFP-tagged protein; Alexa Flour 594: a FMRP antibody fluorescently labeled red. c Quantitative representation of EGFP positive cells. $\mathbf{d}$ Quantitative representation of FMRP positive cells

that BEX1 gene was expressed both in the cytoplasm and cell nucleus, but it was mainly located in the cytoplasm of HEK293T cells, co-localizing with the endogenous FMRP (Fig. 4c\&d).
We performed an integrated bioinformatics analysis on 545 differentially expressed genes (DEGs). Ontological classification revealed that the DEGs were mainly enriched in three categories, including biological process 
A

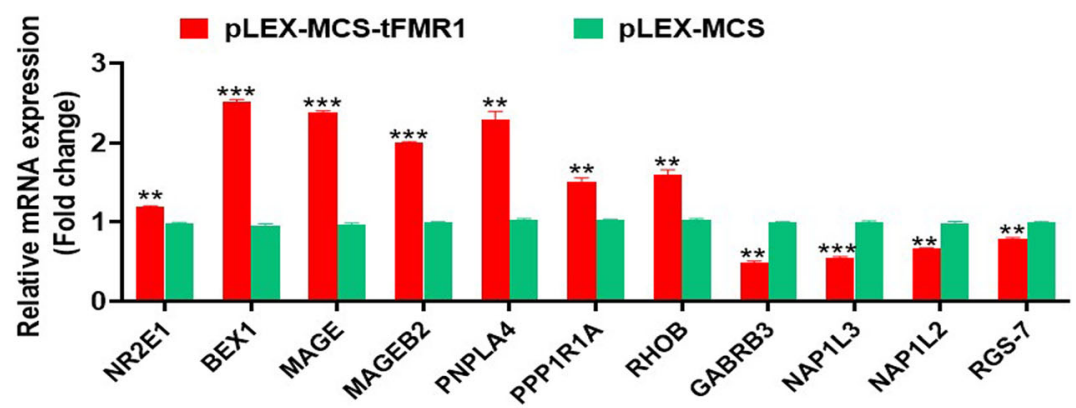

B
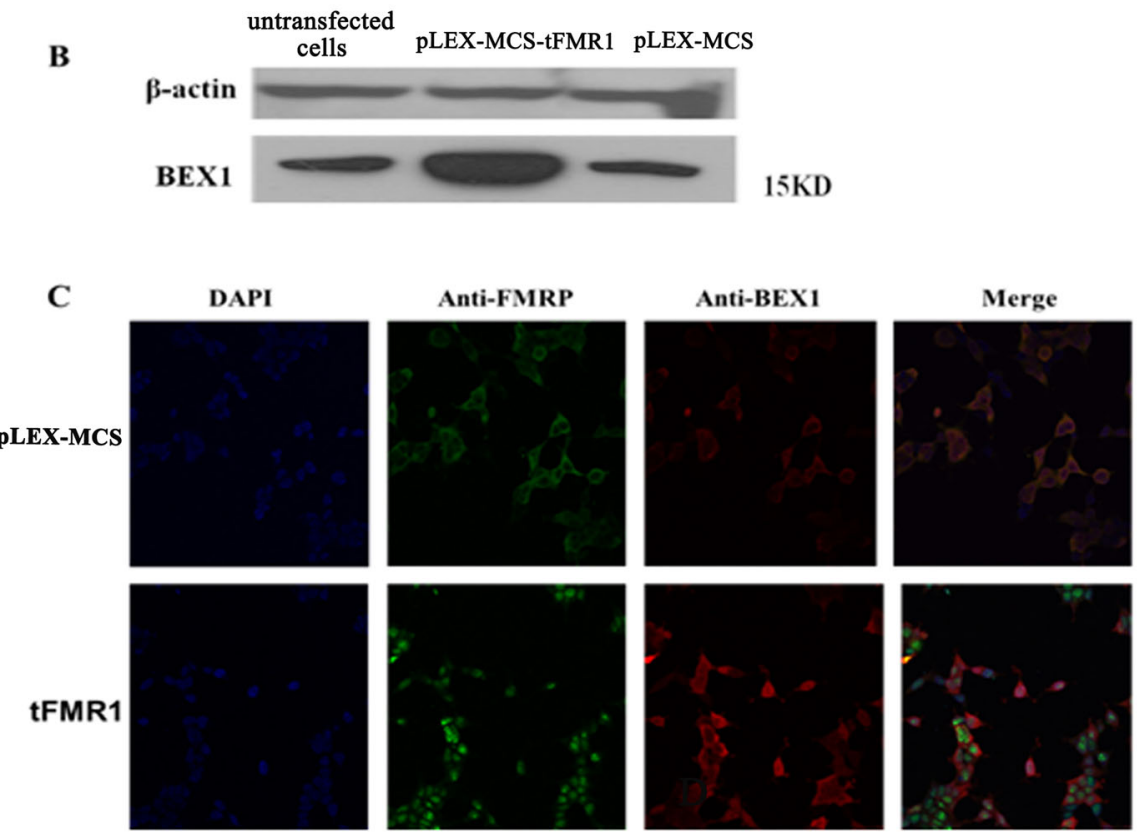

D

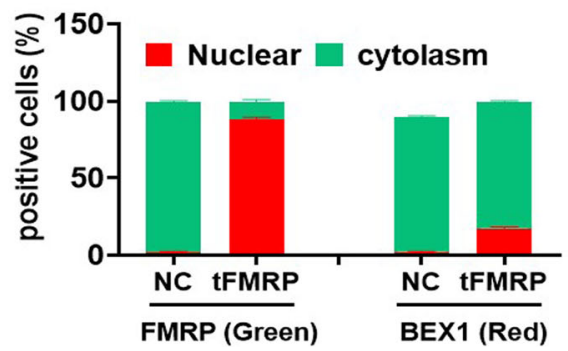

Fig. 4 Overexpression of the truncated FMRP protein altered FXS-related signaling pathways. a Partial qRT-PCR results of differentially expressed genes. Red: HEK293T cells transfected by pLEX-MCS-tFMR1; Green: HEK293T cells transfected by pLEX-MCS. b Western blots showing the overexpression of BEX1 protein in HEK293T cells transfected by pLEX-MCS-tFMR1. 1: untransfected HEK293T cells; 2: HEK293T cells transfected by pLEX-MCS-tFMR1; 3: HEK293T cells transfected by pLEX-MCS. c Immunofluorescence showing the localization of BEX1 protein in HEK293T cells. HEK293T: untransfected HEK293T cells; pLEX-MCS-tFMR1: HEK293T cells transfected by pLEX-MCS-tFMR1. DAPI: the solution suitable for nuclear staining; Anti-FMRP: the primary antibodies of FMRP; Anti-BEX1: the primary antibodies of BEX1. $\mathbf{d}$ Quantitative representation of FMRP and BEX1 positive cells. ${ }^{* *} p<0.01$; ${ }^{* *} p<0.001$

(BP), cellular component (CC), and molecular function (MF). In the BP group, $28 \%$ proteins are concerned with metabolic process and $19 \%$ with cellular process, whereas the DEGs in the CC group comprise of cell part $(37 \%)$, organelle $(25 \%)$, and membrane
(16\%). For the MF group, the DEGs are associated with binding (45\%) and catalytic activity (31\%) (Supplementary Fig. S2C). KEGG analysis demonstrated that the DEGs are mainly involved in four significant signaling pathways, i.e. cancer-related pathways, PI3K- 
Akt, influenza A, and neuroactive ligand-receptor interaction signaling pathways.

\section{Discussion}

Alternative splicing of FMR1 gene

Almost 95\% human genes would experience certain level of alternative splicing (AS) and contribute to proteome complexity [14] AS can also produce mRNAs that differ in their untranslated regions or coding sequence. The mechanisms of AS mainly include exon skipping, intron retention, the use of alternative splice sites, and the choice of mutually exclusive exons. The different spliced isoforms might influence mRNA localization, stability and translation. Moreover, several splicing mRNA variants could alter the reading frame and generate various protein isoforms with diverse localizations and functions. The most common site for AS is in the neural tissue, where various spliced transcripts may function as modulators to synaptic functions. Since the cloning of FMR1 gene as the disease gene of FXS in 1991, a large number of FMR1 mRNAs and FMRP isoforms derived from AS have been detected in mouse and human [15] The distribution of different FMRP isoforms with specific cellular roles and in various tissues is also relatively well understood. It is reported that a high expression level of different FMR1 mRNAs was observed in the brain, testis, placenta, and lymphocytes, whereas a low level of expression was noted in other organs [15] At least 4 predicted FMRP isoforms were identified in mouse brain, which demonstrates that the dissimilar isoforms of FMRP occur together in the same cell type or separately in distinct cell types [16] At present, more than 24 predicted mature transcripts have been reported, and they mainly involve the inclusion or exclusion of exons 12 and 14, and the selection of splice acceptor sites on exons 15 and 17 [17] To our knowledge, our work represents a further study identifying a new exon from intron 9 of human FMR1 gene.

\section{Subcellular localization of FMRP}

The longest isoform of FMRP (isoform 1) is predominantly cytoplasmic and mainly functions as mRNAbinding protein that can directly or indirectly interact with other proteins, regulating the stability of mRNA and maintaining the balance of shuttling between the cytoplasm and nucleus. The nuclear localization signal (NLS) and nuclear export signal (NES) of FMRP are also associated with the cytoplasmic localization of FMRP. A patient with a novel R138Q mutation in the NLS exhibits developmental delay [18] The mechanism of this mutation is unclear, but it may lead to the different distribution of proteins in cytoplasm and nucleus and may indicate the importance of the domain. In addition, exon 14 of FMR1 gene encodes a cytoplasmic retention domain. Exclusion of exon 14 alters the downstream reading frame, generates two different $\mathrm{C}$-terminal regions, and finally results in nuclear localization [7] Furthermore, FMRP C-terminal is one of the determinant factors of nuclear localization and is the key domain that mediates the kinesin and dendrite transmission [19] FMRP homologous proteins, including FXR1 and FXR2, also shuttle between the nucleus and cytoplasm by producing multiple isoforms with different C-terminal [20] Correspondingly, FMRP N-terminal is highly conserved in species [21] Banerjee and his colleagues studied the functional difference among long and short isoforms of FMRP of D. melanogaster, showing that the short isoforms, without the $\mathrm{C}$ terminal region, can easily cause short-term or long-term learning and memory disorders [22]

FMRP can bind key protein cytoplasmic FMRPinteracting protein (CYFIP1), a downstream effector of Rac1 in the cytoplasm, remodeling the cytoskeleton and involving the formation of the translational initiation complex [23] However, previous studies presented that FMRP binds its mRNA targets in the nucleus and facilitates the cargo of nuclear proteins, and the export of FMRP from the nucleus depends on mRNA synthesis. Kim and his colleagues knocked down the mRNA exporter Tap/NXF1, resulting in the increased of FMRP levels in nucleus [24] It also has been proved that FMRP can combine proteins in nucleus, such as NUFIP1 and 82-FIP (FMR1 interacting protein 1), RISC (RNA-induced silencing complex), AGO2, and Dicer (argonaute 2), and eIF5 (eukaryotic translation initiation factor 5), which are pivotal molecules that mediate translational repression by inhibiting the initiation of translation and causing polyribosomes stalling $[25,26]$ Moreover, the combination process of FMRP and ribosomes being an important mechanism of translational regulation occurs in the nucleus. FMRP has been regarded as chromatinassociated protein. This protein can coimmunoprecipitate with chromatin as confirmed by Chip sequencing, and by interacting with nucleolin, affecting the transcription of rRNA and the biosynthesis of ribosomes [27]

Given the lack of nuclear export signal, our newly identified FMRP isoform with a short C-terminal retains in the nucleus. We speculate that the increase in FMRP alternatively spliced isoforms in the nucleus could disrupt the balance of shuttling between the nucleus and cytoplasm and affect the translational repression of FMRP.

\section{New interactors of FMRP in the FXS-related signaling pathways}

With the development of several high-throughput approaches, such as microarray analysis, HITS-highthroughput sequencing of RNA isolated by crosslinking immunoprecipitation (CLIP) and PAR-photoactivatable ribonucleoside-enhanced CLI P (PAR-CLIP), research 
revealed that FMRP can interact with about 5\% mRNA targets in the brain [4] FMRP is a translational repressor involved in the regulation of synaptic functions via the activation of NMDA receptors, AMPA receptors and GABA receptors, which contribute to the formation of long-term depression (LTD) and long-term potentiation (LTP), according to mGluR theory [28] It is widely believed that the regulation of neurological function mainly depends on the mGluR-LTD pathway, which mediates the synaptic plasticity and hinges on the local protein synthesis of dendrites. The mGluR theory of FXS emphasizes that FMRP is downstream of mGluRs and upstream of local protein synthesis. FMRP has been suggested to repress the translation of dendritically localized mRNAs. With the activation of mGluR, FMRP repression would allow the synthesis of local protein in response to synaptic stimulation, resulting in the AMPAR internalization and LTD [28] For patients with FXS, the absence of FMRP could constructively increase protein synthesis, leading to the over activation of AMPAR internalization and LTD exaggeration. The extracellular signal-related kinase (ERK) and mammalian target of rapamycin (mTOR) signaling pathways are required for the regulation of mGluR-LTD $[29,30]$ Studies have shown that antagonizing the mGluR pathway can alleviate the phenotypes of FXS [31] Therefore, mGluR theory provides new avenues for the understanding of pathological mechanisms and therapeutic intervention of FXS.

Interestingly, our findings in RNA microarray analysis revealed GABRB3 and BEX1 as the most significantly down-regulated and up-regulated genes, respectively (Supplementary Fig. S2). Altered expression of mRNA and protein for GABA receptors has been reported in FMR1 knockout mice, implying that the loss of FMRP can affect GABA receptor subunit expression. Recent publications have also identified that the absence of FMRP can cause the upregulation of mGluR signaling, resulting in the decreased expression of GABRB3 protein, consistent with our RNA microarray results [3234] Therefore, the overexpression of truncated FMRP protein has profound effects on FMRP-mGluR-GABRB3 signaling pathway. BEX1 gene may also participate in mGluR-LTD and mGluR-LTP signaling pathways. BEX1 gene is linked to neurotrophin signaling as an interactor of the Trk tyrosine kinases (TrkA, TrkB and TrkC) or p75 neurotrophin receptor (p75NTR), regulating differentiation, growth, and survival of neuronal and glial cells [35] Trk receptors can be activated by several canonical pathways, including the phosphatidylinositol-3 kinase (PI3K)/AKT/mTOR and Ras/MAP kinase signaling pathways [36] Additionally, TrkB can participate in the LTP signaling pathways and mediate the synaptic plasticity [37] However, the signaling mechanisms of p75NTR are still poorly understood. p75NTR can change the functions of the amygdale [38] and contribute to multiple cell responses processes, such as apoptosis, survival, axonal growth, and cell death. When BEX1 protein is overexpressed, it can inhibit the NF- $\mathrm{kB}$ activity by Trk receptors and $\mathrm{p} 75 \mathrm{NTR}$, without affecting the activation of AKT and Erk1/2 signaling [35] which are critical molecules involved in mGluR-LTD signaling pathways. The high BEX1 level in connection with the mGluR-LTP or mGluR-LTD signaling provides new insights into the interactions of FMRP in FXS-related signaling pathways.

\section{Conclusion}

In conclusion, our study identified a new exon from amid intron 9 of human FMR1 gene with wide expression in normal healthy individuals. In particular, sequences similar to the new exon can be only found in genomes of primates, and its insertion can produce a truncated FMRP with altered cellular localization. Our preliminary data from RNA microarray analysis points to the possibility that $B E X 1$ gene may be a new player in the FXS-related mGluR-LTP or mGluR-LTD signaling pathways. However, the complicated molecular mechanisms of this new alternative exon-influenced roles of FMRP await further clarification.

\section{Methods}

RNA isolation and CDNA synthesis materials

Peripheral blood samples were obtained from the Department of Laboratory Medicine of 900th Hospital of the Joint Logistics Force. RNA was extracted from the peripheral blood of 52 non-FXS individuals using RNA isolation kit (Qiagen, Hilden, Germany), following the manufacturer's protocol and stored at $-80^{\circ} \mathrm{C}$ for subsequent use. The quality of RNA was evaluated by Biophotometer (Eppendorf, Hamburg, Germany). Synthesis of the cDNA was carried out following the instructions of the manufacturer (Toyobo, Osaka, Japan).

\section{Real-time reverse transcriptase PCR}

qRT-PCR reactions were performed using iTaq SYBR Green Kits followed the 3-step cycles from the manufacturer's protocol (Toyobo, Osaka, Japan). All reactions were carried out in triplicates and the cycles were run on Bio-Rad CFX96 real-time system (Bio-Rad, Hercules, CA, USA). The primers used were listed in Supplementary Tab. S1.

\section{Bioinformatics}

Four softwares were used to evaluate the splicing signals in this study, including ASD (http://www.ebi.ac.uk/asd), HSF (http://www.umd.be/HSF/), BDGP (http://www. fruitfly.org/seq_tools/splice.html), and ASPicDB (http://t. caspur.it/ASPicDB/index.php). 


\section{Western blot}

We used $10 \%$ polyacrylamide gel to separate target proteins, which were extracted from cell lysates using a lysis buffer $(50 \mathrm{mM}$ Tris- $\mathrm{HCl}, \mathrm{pH} 7.4,150 \mathrm{mM} \mathrm{NaCl}, 1 \%$ Triton $\mathrm{X}-100,1 \%$ sodium deoxycholate, $0.1 \%$ SDS and protease inhibitor mixture). The protein concentration was determined by bicinchoninic acid assay protein assay (Bio-Rad, Hercules, CA, USA). The proteins were transblotted onto polyvinylidene fluoride membrane (BioRad, Hercules, CA, USA), and the membrane was blocked with Tris-buffered saline (TBS) containing 5\% non-fat milk for $1 \mathrm{~h}$, and incubated with a mouse monoclonal antibody anti-FMRP (Abcam, Cambridge, MA, USA) at 1:750 dilution for overnight incubation at $4{ }^{\circ} \mathrm{C}$. On the next day, the membrane was incubated with a horseradish peroxidase (HRP)-conjugated secondary antibody (Santa Cruz, Dallas, Texas, USA) at 1:5000 dilution for $1 \mathrm{~h}$. The membrane was washed thrice, and enhanced chemiluminescence solution (ECL) (Beyotime, Shanghai, China) was added while exposing the film in accordance with conventional procedures. For the detection of BEX1 protein, a rabbit monoclonal anti-BEX1 (Abcam, Cambridge, MA, USA) primary antibody at 1:1000 and HRPconjugated anti-rabbit secondary antibody were used.

\section{Plasmids construction}

HEK293T cells (Catalog Number: GNHu17) were obtained from Shanghai Cell Bank, Chinese Academy of Science. To identify the subcellular distribution of the end product encoded by the novel transcript containing the $140 \mathrm{bp}$ sequence, we inserted the full-length coding sequence of FMR1 or fragment containing exons 1-9 and the $140 \mathrm{bp}$ sequence into the eukaryotic expression vector pEGFP-N2 (BD Biosciences, San Jose, CA, USA). Fulllength coding sequence of FMR1 gene was amplified with primers wFMR1-F and wFMR1-R (wFMR1-F: 5' $-A A A-$ GAGCTCGATGGAGGAGCTGGTGGTGGA AG-3'; wFMR1-R:5' -ACGCGCGACCGGGTACTCCATTCACG

AGTG-3';), whereas the fragment containing exons 1-9 and the $140 \mathrm{bp}$ sequence was amplified with primers tFMR1-F and tFMR1-R (tFMR1-F: 5'-AAAGAGCTCG ATGGAGGAGCT GGTGGTGGAAG-3'; tFMR1-R:5' GCGTCGACCGACTTCAACCCTACTAAGT

TCCTTGGA-3'). All primers contained the restrictive enzyme sites Sac Iand Sal I, for convenience of subcloning. For construction of the lentiviral overexpression vector, the target coding fragment was amplified using specific primers tFMR1-PF and tFMR1-PR with Not Iand $M l u$ Isites, respectively (tFMR1-PF: 5' -AAAT ATGCGGCCGC ATGGAGGAGCTGGTGGTGGAA-3'; tFMR1-PR: 5' GCGACGC GTTCAGATCTTCAACCCTACTA A-3'). After the amplified product was linked to the lentiviral vector pLEX-MCS, the packaging plasmids pMD2.G and psPAX2 (Open Biosystems, Huntsville, USA) were used to co-transfect HEK293T cells using polyethylenimine (PEI) reagent (Sigma, St. Louis, MO, USA).

\section{Cell culture and transfection}

HEK293T cells (Catalog Number: GNHu17) were cultured in Dulbecco's Modified Eagle Medium (DMEM) medium with $10 \%$ fetal bovine serum (FBS) and $1 \%$ penicillin/streptomycin (Invitrogen, Carlsbad, CA, USA). HEK293T cells were transfected with the recombinant eukaryotic vectors using Lipofectamine 2000 (Invitrogen, Carlsbad, CA, USA) following the manufacturer's instructions. Three days later, the cells were observed, and RNA/ protein was extracted for subsequent use. As for the recombinant lentiviral vector, transfection of HEK293T cells followed the recommended protocol (Sigma, St. Louis, MO, USA) by use of PEI reagent. The virus supernatant of cells was collected per $24 \mathrm{~h}$ for 3 days and used to infect a new batch of HEK293T cells. The newly infected HEK293T cells were cultured in DMEM/F12 (1,1) medium with puromycin for about 15 days. Finally, stably transfected HEK293T cells were harvested.

\section{Immunofluorescence}

The subcellular distribution of proteins was examined by immunofluorescence staining. Specifically, the cells were fixed with $4 \%$ paraformaldehyde for $30 \mathrm{~min}$, permeabilized with $0.5 \%$ Triton X-100 for $20 \mathrm{~min}$, and incubated with $3 \%$ bovine serum albumin (BSA) for $1 \mathrm{~h}$. Then, the cells were stained with the primary antibodies of interest, such as anti-FMRP and anti-BEX1 (Abcam, Cambridge, MA, USA) overnight at $4{ }^{\circ} \mathrm{C}$. On the next day, the cells were washed thrice with phosphatebuffered saline (PBS) and incubated with secondary antibodies Alexa Fluor ${ }^{\circledR}$ 594-conjugated goat anti-mouse IgG or goat anti-rabbit IgG for $2 \mathrm{~h}$ at room temperature (Santa Cruz, Dallas, Texas, USA). The cell nuclei were stained with 4',6-diamidino-2-phenylindole (DAPI) dye for $5 \mathrm{~min}$ (Beyotime, Shanghai, China). Finally, we observed cells under an FV1000 laser-scanning confocal microscope (Olympus, Tokyo, Japan).

\section{RNA microarray analysis}

Total RNA was extracted from two batches of stably transfected HEK293T cells, including HEK293T cells transfected by void lentiviral vector (pLEX-MCS) and those by pLEX-MCS-tFMR1 vector. After the evaluation of RNA quality according to manufacturer's protocol, RNA microarray hybridization was performed at Capital Bio Company (Beijing, China) and Affymetrix Human Genome U133 Plus 2.0 was used for analysis (Affymetrix, Santa Clara, CA, USA). For the functional analysis of the differentially expressed genes, DAVID (http://david.abcc.ncifcrf.gv/) database was also used. 


\section{Statistical analysis}

All experimental data were expressed as means \pm SEM. Data were analyzed using $t$-test by using SPSS 18.0. Differences were considered statistically significant at $p<$ 0.05 in all cases.

\section{Accession numbers}

Insertion sequence of FMR1 gene is available from GenBank MF593118. Sequenced reads have been deposited in the NCBI Gene Expression Omnibus (GEO) database (accession number GSE101830).

\section{Supplementary information}

Supplementary information accompanies this paper at https://doi.org/10. 1186/s12863-020-00870-2.

Additional file 1: Figure S1. Comparative genomics showed that sequences homologous to the $140 \mathrm{bp}$ sequence are only found in the genomes of primates. Multiple sequences alignment of FMR1 in mammals. The part underlined in green represents the140 bp novel alternative splice exon and its splicing signals, and the red square indicates the 140 bp novel alternative splice exon. Figure S2. RNA microarray analysis of overexpressed truncated FMRP protein and ontological classification of differentially expressed genes. Table S1. Sequence of qRT-PCR primers. Table S2. Top 20 up-regulated expression genes in HEK293T cells with overexpressed tFMRP protein. Table S3. Top 20 down-regulated expression genes in HEK293T cells with overexpressed tFMRP protein

\section{Acknowledgements}

We thank Dr. Kuancan Liu for pLEX-MCS lentiviral Vector and for technical assistance.

\section{Authors' contributions}

$F L$ and $W Y$ conceived and designed the investigation. WY and AY carried out most of the experiments. $X G$ and $X F$ carried out bioinformatics analyses. $Y X, J L, D L$, and DZ performed some of the experiments. WY, YX wrote the manuscript and FL revised it. All authors have read and approved the manuscript.

\section{Funding}

This work was supported by Key Scientific Projects of Fujian Province, P. R. China (2010Y0045, 2016Y0071, for sampling costs, to support data analysis and interpretation), Fujian Provincial Natural Science Foundation of China (2018 J01341, for data analysis and interpretation, and for manuscript preparation), and an innovation team grant from 900th Hospital of the Joint Logistics Force (for reproductive health, for data analysis and manuscript preparation)

\section{Availability of data and materials}

The datasets used and/or analyzed during the current study are available from the corresponding author on reasonable request.

\section{Ethics approval and consent to participate}

Not applicable.

\section{Consent for publication}

Not applicable.

\section{Competing interests}

The authors declare that they have no conflict of interest.

\section{Author details}

'Department of Clinical Genetics and Experimental Medicine, 900th Hospital of the Joint Logistics Force, Xiamen University School of Medicine, 156 Xi'erhuanbei Road, Fuzhou City, Fujian Province 350025, People's Republic of
China. ${ }^{2}$ Present addresses: Department of Laboratory Medicine, Zhongshan Hospital, Fudan University, Shanghai 200032, China. ${ }^{3}$ Present addresses: Department of Laboratory Medicine, Fuzhou No. 2 Hospital Affiliated Xiamen University, Fuzhou, Fujian 350007, People's Republic of China. ${ }^{4}$ Present addresses: Department of Laboratory Medicine, Ningde Municipal Hospital, Fujian Medical University, Ningde City 352100, Fujian Province, China. ${ }^{5}$ Present addresses: Department of Laboratory Medicine, Fujian University of Traditional Chinese Medicine Affiliated People's Hospital, Fuzhou 350001, Fujian, China.

Received: 7 January 2020 Accepted: 9 June 2020

Published online: 18 June 2020

\section{References}

1. Colak D, Zaninovic N, Cohen MS, Rosenwaks Z, Yang WY, Gerhardt J. Promoter-bound trinucleotide repeat mRNA drives epigenetic silencing in fragile X syndrome. Science. 2014;343(6174):1002-5.

2. Adams-Cioaba MA, Guo Y, Bian C, Amaya MF, Lam R. Structural studies of the tandem Tudor domains of fragile $X$ mental retardation related proteins FXR1 and FXR2. PLoS One. 2010:5:e13559.

3. Alpatov R, Lesch BJ, Nakamoto-Kinoshita M, Blanco A, Chen S, Stützer A, Armache KJ, Simon MD, Xu C, Ali M, Murn J, Prisic S, Kutateladze TG, Vakoc CR, Min J, Kingston RE, Fischle W, Warren ST, Page DC, Shi Y. A chromatindependentrole of the fragile $X$ mental retardation protein FMRP in the DNA damage response. Cell. 2014;157(4):869-81.

4. Dolzhanskaya N, Sung YJ, Conti J, Currie JR, Denman RB. The fragile $X$ mental retardation protein interacts with $U$-rich RNAs in a yeast threehybrid system. Biochem Biophys Res Commun. 2003;305:434-41.

5. Halevy T, Czech C, Benvenisty N. Molecular mechanisms regulating the defects in fragile $X$ syndrome neurons derived from human pluripotent stem cells. Stem Cell Rep. 2015;4(1):37-46.

6. Wang H, Wu LJ, Kim SS. FMRP acts as a key messenger for dopamine modulation in the forebrain. Neuron. 2008:59(4):634-47.

7. Sittler A, Devys D, Weber C, Mandel JL. Alternative splicing of exon 14 determines nuclear or cytoplasmic localisation of FMR1 protein isoforms. Hum Mol Genet. 1996;5(1):95-102.

8. Raymond CK, Castle J, Garrett-Engele P, Armour CD, Kan Z. Expression of alternatively spliced sodium channel alpha-subunit genes. Unique splicing patterns are observed in dorsal root ganglia. J Biol Chem. 2004;279:4623441.

9. Loffreda A, Rigamonti A, Barabino SM, Lenzken SC. RNA-binding proteins in the regulation of miRNA activity: a focus on neuronal functions. Biomolecules. 2015;5(4):2363-87.

10. Chetkovich DM, Bunn RC, Kuo SH, Kawasaki Y, Kohwi M. Postsynaptic targeting of alternative postsynaptic density-95 isoforms by distinct mechanisms. J Neurosci. 2002:22:6415-25.

11. Raingo J, Castiglioni AJ, Lipscombe D. Alternative splicing controls $G$ protein-dependent inhibition of $\mathrm{N}$-type calcium channels in nociceptors. Nat Neurosci. 2007;10:285-92.

12. Guo X, Fu X, Yan A, Liao J, Zhang D, Ke L, Lan F. Identification of a novel cryptic exon in intron 9 region of FMR1 gene among fragile $X$ syndrome (article in Chinese). Int J Lab Med. 2013;34(1):6-8.

13. Tseng E, Tang H-T, AlOlaby RR, Hickey L, Tassone F. Altered expression of the FMR1 splicing variants landscape in permutation carriers. BBA-Gene Regulatory Mechanisms. 2017;1860:1117-26.

14. Pan Q, Shai O, Lee $L J$, Frey BJ, Blencowe BJ. Deep surveying of alternative splicing complexity in the human transcriptome by high-throughput sequencing. Nat Genet. 2008:40:1413-5.

15. Verkerk AJ, de GE, De BK, Eichler EE, Konecki DS, Reyniers E, Manca A, Poustka A, Willems PJ, Nelson DL. Alternative splicing in the fragile X gene FMR1. Hum Mol Genet. 1993;2:399-404.

16. Xie W, Dolzhanskaya N, LaFauci G, Dobkin C, Denman RB. Tissue and developmental regulation of fragile $X$ mental retardation 1 exon 12 and 15 isoforms. Neurobiol Dis. 2009;35(1):52-62.

17. Pretto DI, Eid JS, Yrigollen CM, Tang HT, Loomis EW, Raske C, DurbinJohnson B, Hagerman PJ, Tassone F. Differential increases of specific FMR1 mRNA isoforms in premutation carriers. J Med Genet. 2015:52(1):42-52.

18. Collins SC, Bray SM, Suhl JA, Cutler DJ, Coffee B, Zwick ME, Warren ST. Identification of novel FMR1 variants by massively parallel sequencing in developmentally delayed males. Am J Med Genet A. 2010;152A(10):2512-20. 
19. Pilaz $L$, Lennox AL, Rouanet JP, Silver DL. Dynamic mRNA transport and LocalTranslation in radial glial progenitors of the developing brain. Curr Biol. 2016;26(24):3383-92.

20. Tamanini F, Kirkpatrick LL, Schonkeren J, van Unen. L, Bontekoe C, Bakker C, Nelson DL, Galjaard H, Oostra BA, Hoogeveen AT. The fragile X-related proteins FXR1P and FXR2P contain a functional nucleolar-targeting signal equivalent to the HIV-1 regulatory proteins. Hum Mol Genet. 2000;9:148793.

21. Schenck A, Bardoni B, Moro A, Bagni C, Mandel JL. A highly conserved protein family interacting with the fragile $X$ mental retardation protein (FMRP) and displaying selective interactions with FMRP-related proteins FXR1P and FXR2P. Proc Natl Acad Sci U S A. 2001;98:8844-9.

22. Banerjee P, Schoenfeld BP, Bell AJ, Choi CH, Bradley MP. Short- and longterm memory are modulated by multiple isoforms of the fragile $X$ mental retardation protein. J Neurosci. 2010:30:6782-92.

23. Bonaccorso CM, Spatuzza M, Di Marco. B, Gloria A, Barrancotto G, Cupo A, Musumeci SA, D'Antoni S, Bardoni B, Catania MV. Fragile X mental retardationprotein (FMRP) interacting proteins exhibit different expression patterns during development. Int J Dev Neurosci. 2015;42:15-23.

24. Kim M, Bellini M, Ceman S. Fragile X mental retardation protein FMRP binds mRNAs in the nucleus. Mol Cell Biol. 2009;29(1):214-28.

25. Ceman S, Brown V, Warren ST. Isolation of an FMRP-associated messenger ribonucleoprotein particle and identification of nucleolin and the fragile $X$ related proteins as components of the complex. Mol Cell Biol. 1999:19: 7925-32.

26. El Fatimy. R, Davidovic L, Tremblay S, Jaglin X, Dury A, Robert C, De Koninck. $P$, Khandjian EW. Tracking the fragile $X$ mental retardation protein in a highly ordered neuronal RiboNucleoParticles population: a link between stalled polyribosomes and RNA granules. PLoS Genet. 2016;12(7):e1006192.

27. He Q, Ge W. FMRP: a new chapter with chromatin. Protein Cell. 2014;5(12): 885-8.

28. Bear MF, Huber KM, Warren ST. The mGluR theory of fragile $X$ mental retardation. Trends Neurosci. 2004:27:370-7.

29. Narayanan U, Nalavadi V, Nakamoto M, Thomas G, Ceman S. S6K1 phosphorylates and regulates fragile $\mathrm{X}$ mental retardation protein (FMRP) with the neuronal protein synthesis-dependent mammalian target of rapamycin (mTOR) signaling cascade. J Biol Chem. 2008:283:18478-82

30. Kim SH, Markham JA, Weiler IJ, Greenough WT. Aberrant early-phase ERK inactivation impedes neuronal function in fragile $X$ syndrome. Proc Natl Acad Sci U S A. 2008;105:4429-34.

31. Darnell JC, Klann E. The translation of translational control by FMRP: therapeutic targets for FXS. Nat Neurosci. 2013;16:1530-6.

32. Provenzano G, Sgadò P, Genovesi S, Zunino G, Casarosa S, Bozzi Y Hippocampal dysregulation of FMRP/mGluR5 signaling in engrailed-2 knockout mice: a model ofautism spectrum disorders. Neuroreport. 2015; 26(18):1101-5.

33. Fatemi SH, Folsom TD, Kneeland RE, Liesch SB. Metabotropic glutamate receptor 5 upregulation in children with autism is associated with underexpression of both fragile $X$ mental retardation protein and GABAA receptor beta 3 in adults with autism. Anat Rec. 2011;294:1635-45.

34. Hong A, Zhang A, Ke Y, El Idrissi. A, Shen CH. Downregulation of GABA(a) $\beta$ subunits istranscriptionally controlled by FMR1p. J Mol Neurosci. 2012;46(2): $272-5$.

35. Vilar M, Murillo-Carretero M, Mira H, Magnusson K, Besset V, Ibáñez CF. Bex1, a novel interactor of the p75 neurotrophin receptor, links neurotrophin signaling to the cell cycle. EMBO J. 2006;25(6):1219-30.

36. Huang EJ, Reichardt LF. TRK receptors: roles in neuronal signal transduction. Annu Rev Biochem. 2003;27:27.

37. Leal G, Bramham CR, Duarte CB. BDNF and hippocampal synaptic plasticity. Vitam Horm. 2017;104:153-95.

38. Busch, R., Baldus, M., Vogt, M. A., Berger, S. M., Bartsch, D, Gass, P., von, Bohlen, Und, Halbach. O. (2017) Effects of p75NTR deficiency on cholinergic innervation of the amygdala and anxiety-like behavior. J Neurochem..

\section{Publisher's Note}

Springer Nature remains neutral with regard to jurisdictional claims in published maps and institutional affiliations.

\section{Ready to submit your research? Choose BMC and benefit from:}

- fast, convenient online submission

- thorough peer review by experienced researchers in your field

- rapid publication on acceptance

- support for research data, including large and complex data types

- gold Open Access which fosters wider collaboration and increased citations

- maximum visibility for your research: over $100 \mathrm{M}$ website views per year

At BMC, research is always in progress.

Learn more biomedcentral.com/submissions 